Chapter 4

\title{
Advanced Human In vitro Models for the Discovery and Development of Lung Cancer Therapies
}

\author{
Samuel Constant, Song Huang, \\ Ludovic Wisniewski and Christophe Mas \\ Additional information is available at the end of the chapter \\ http://dx.doi.org/10.5772/60606
}

\section{Introduction}

Lung cancer is the leading cause of cancer deaths in both men and women, with more than 1 million deaths worldwide each year [1]. Unfortunately, this difficult therapeutic area has shown the highest failure rate in clinical trials over the last 30 years [2] and hitherto, there is no effective treatment for patients with lung cancer. The reasons for this drug attrition are multiple, but one major explanation is considered to be the lack of relevant preclinical models to appropriately validate potential drug targets and rank novel therapeutic agents before engaging in clinical trials [3].

Genetically engineered mice, ectopic and orthotopic xenotransplantation of tumors into immunodeficient mice are common models used as surrogate of patients to evaluate drug candidates before clinical testing. Although animal models can recapitulate important facets of human responses, their limitations as preclinical cancer models have now been widely documented [4-7]. Fundamental differences in transcriptional regulation [8], telomerase activity [9], neoplastic transformation mechanisms [10], cytokines production [11] as well as matrix metalloproteinases biology [12] are but a few features which compromise the design of efficient cancer therapies. Even the patient derived xenograft model (PDX), which better recapitulate the phenotypic features of the human tumor, displays a number of inherent limitations [13]. In this system, the tumorgraft established from primary tumor fragments has to be maintained through serial transplantations into mice, which will lead to the loss of the human stroma environment after 2-3 passages [14]. Clearly, such a replacement of the original tumor microenvironment by murine host components has significant consequences on growth features as well as response to therapies. Indeed, a number of oncogenic mouse ligands fail to cross-activate their related human receptors $[15,16]$ while stromal mediators have been 
identified as a critical source triggering tumour cells resistance to treatment $[17,18]$. These observations highlight the importance of considering tumor-extracellular matrix interactions in the design of in vitro cancer models. Modern tumor biology has moved away from the traditionalist view conveyed for years by $2 \mathrm{D}$ cultures saturated with growth factors by revealing that the many varieties of cells that compose a tumor don't just grow on their own but constantly integrate and react to signals coming from the extracellular matrix components. Solid tumors are now regarded as complex organs able to instruct the surrounding tissue to promote their own growth and progression, but also dependent on both molecular and mechanical signals coming from the adjacent healthy environment $[19,20]$. Therefore, experimental models recapitulating true human cancer biology are mandatory for the validation of therapeutic agents in order to keep away from the risk of studying no more than adaptive cancer biology in a wrong environment that will eventually result in translation failure.

A

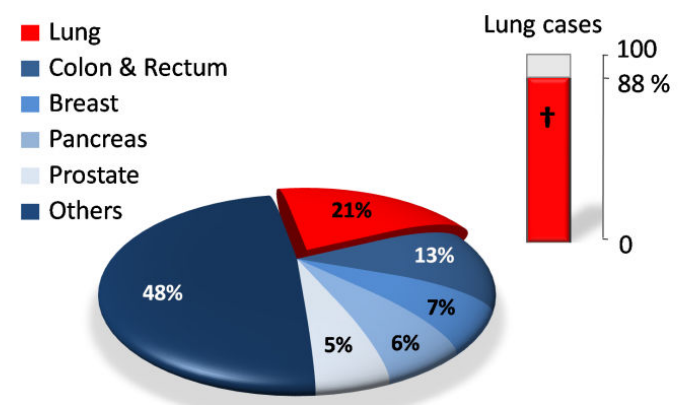

$\mathrm{B}$

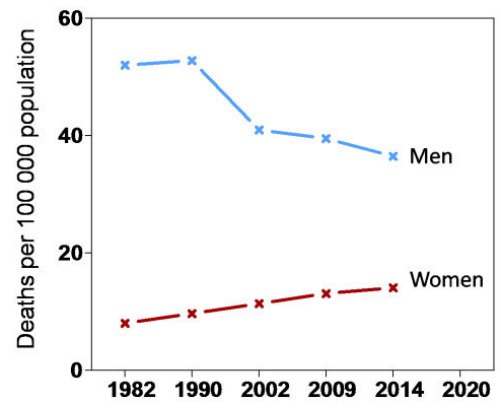

Figure 1. (A) Cancer deaths anticipated in 2014. Estimated leading cancer sites mortality in European Union for the year 2014 expressed as percent of total cancer deaths. Column diagram highlights the mortality rate within the population specifically affected by lung cancer. (B) Age-standardized (world population) EU male (blue) and female (red) lung cancer death rates per 100,000 from 1982 to 2014. The graph shows the unfavourable trend for female lung cancer with a regular increase in case numbers over the last 30 years. Source: Malvezzi et al, Annals of oncology, 2014; 25(8): 1650-6. and Bosetti et al, Annals of oncology, 19: 631-640, 2008.

In this article we report the significant efforts ongoing within academia and industry to developing in vitro novel complex human tumor models that should improve the identification and selection of efficient lung cancer therapies. We first focus on the human lung cancer cell lines currently available, their contribution to lung cancer biology and their use in research. Then, we will move from cell monolayers to three-dimensional (3D) cultures, exploring at first the function of natural and synthetic extracellular matrix before to document some recent advances in the field, including spheroids, bioscaffolds, decellularized lung matrix models and precision-cut lung tumor slices. Finally, we will present the bioengineering of a new generation of lung cancer models: OncoCilAir ${ }^{\mathrm{TM}}$. These $100 \%$ human models, which combine in vitro both tumor nodules surrounded by a functional airway epithelium and stroma, open new ways to test simultaneously drug efficacy, side toxicity and tumor recurrence in a single, integrated and accessible 3D model. 


\section{Lung cancer, current treatments and perspectives}

Lung cancer (LC) is one of the major health concerns in the western world. LC is the most frequently diagnosed cancer in men and women and represents the most common cause of cancer-related deaths, both in the United States and in Europe, with a significant rate of $27 \%$ and $21 \%$ of total cancer deaths, respectively [21] (Fig.1A). The LC epidemic has been clearly linked to tobacco smoking [22], and while mortality rate in men has regularly fallen over the last decades (53 lung cancer deaths for every 100,000 European males in the late 1980s to $41.1 / 100,000$ in 2009) thanks to strong measures for smoking control and prevention in middleaged men, female LC rates are predicted to rise $8 \%$ in 2015 [1] (Fig.1B). There are two main types of lung cancer: non-small lung cancer (NSCLC) which account for about $85 \%$ of all lung cancers and small cell lung cancer (SCLC, 15\%). SCLC is the most aggressive form of LC, with fast growing cells leading to large tumors. Histologically, NSCLC includes 3 subgroups: adenocarcinoma, squamous cell and large cell carcinoma [23]. As in many other forms of cancer, LC does not display too many symptoms, develops slowly over a period of several years, and only manifests itself in advanced stages (III or IV), where five-year survival rates are less than $10 \%$ because of high degree of metastasis. The overall median survival in stage IV is only about 8-10 months [24]. Platinum and taxane based chemotherapies (cisplatin, paclitaxel) has remained for years the treatments of choice, but more recently LC patients have been selected based on their tumor mutation profile. In most cases, oncogene driver mutations are exclusives (EGFR, ALK/EML4, KRAS, PTEN, etc...) and importantly, they divided patient populations into molecular subsets that do not show the same sensitivity to different treatments [25]. This patient stratification has enabled the introduction of targeted therapies directed against specific signaling pathways whose tumors are dependent on. Indeed, humanized recombinant antibodies directed against the vascular endothelial growth factor (VEGF) (bevacizumab) or small molecule inhibitors of EGFR-TK (erlotinib, gefitinib and afatinib), ALK and MET (crizotinib, ceritinib) have recently been used as a promising new line of therapies to treat lung cancer. Unfortunately, this drug portfolio extends survival only by a few months (Table 1) since most of the patients develop resistance to treatments, leading invariably to the recurrence of the disease [26-32]. Huge efforts are now undertaken to understand and circumvent drug resistance mechanisms. First observations have pointed out two main mechanisms for drug resistance acquisition: the selection of another pre-existing oncogene mutation or the activation of a bypass track, i.e. the deregulation of an alternative growth signaling pathway to maintain cell proliferation and tumor progression [33]. In EGFR-mutant lung cancer treated with the EGFR-TK inhibitors erlotinib and gefitinib, resistance is generally mediated by the T790M EGFR second-site mutation ( $50 \%$ of the cases) [34] or phosphatidylinositol 3-kinase PI3K-AKT pathway activation via focal amplification of MET as second signaling pathway ( 5\%) [35]. For $A L K$-mutant lung cancer treated with crizotinib, mechanisms of resistance include the gatekeeper mutation L1196M ( $\sim 30 \%$ of the cases) and KIT and EGFR signaling pathways activation as bypass track ( 45\%) [36]. Overall, these findings argue for the use of combined therapies in a manageable way. But recent data based on the analysis of tumor specimens at the time of acquired resistance suggest a much more complex landscape. In fact, multiple mechanisms, as diverse as epigenetic changes [37], 
epithelial to mesenchyme transition (EMT) or conversion from a LC histological type to another (EGFR-dependent NSCLC to SCLC), are induced under the selective pressure of targeted therapies $[38,39]$. These observations imply the arrival of a personalized medicine, where a careful profiling of patient tumor's mutation status (germline and somatic) and epigenetic signature will be mandatory all along the therapy to identify and adapt the correct treatment strategy. However, such scheduled combinatorial regimen would require the development of multiple-generation inhibitors to overcome specific subsets of resistance mutations and induce durable remissions. But the ability to escape multiple types of treatment could well be a hallmark of cancer cells. With this in mind, alternative strategies are worth considering. Instead of constraining tumor cells signalling through exogenous therapies making them overreact, a different approach might be to restrict tumor progression through its own microenvironment. The original 1975's experiment of teratocarcinoma injection into blastocysts from Mintz and Illmensee was the first example of tumor repression by the microenvironment [40]. Today, tumor reprogramming through stroma instruction is emerging as a new treatment paradigm [41-43]. From this perspective, advanced human three-dimensional (3D) cell culture approaches modelling tumor-stroma communication could be key to accelerate the development of new lung cancer therapeutics.

\begin{tabular}{|c|c|c|c|c|c|c|c|}
\hline Generic & $\begin{array}{l}\text { Trade } \\
\text { Name }\end{array}$ & Indication & Type & Class & Target & PFS & Ref \\
\hline Afatinib & Gilotrif & NSCLC & $\begin{array}{c}\text { small } \\
\text { molecule }\end{array}$ & $\begin{array}{c}\text { antineoplastic } \\
\text { antiangiogenesis }\end{array}$ & $\begin{array}{c}\text { EGFR } \\
\text { HER2-4 }\end{array}$ & $3.3-13.6$ & [32] \\
\hline Crizotinib & Xalkori & NSCLC & $\begin{array}{c}\text { small } \\
\text { molecule }\end{array}$ & antineoplastic & $\begin{array}{c}\text { ALK } \\
\text { MET, ROS1 }\end{array}$ & 9.7 & [29] \\
\hline Ceritinib & Zykadia & NSCLC & $\begin{array}{c}\text { small } \\
\text { molecule }\end{array}$ & antineoplastic & $\begin{array}{c}\text { ALK } \\
\text { IGF1R }\end{array}$ & 7 & [31] \\
\hline Erlotinib & Tarceva & NSCLC & $\begin{array}{c}\text { small } \\
\text { molecule }\end{array}$ & $\begin{array}{c}\text { antineoplastic } \\
\text { antiangiogenesis }\end{array}$ & EGFR & $12-14$ & {$[26 ; 27]$} \\
\hline Gefitinib & Iressa & NSCLC & $\begin{array}{c}\text { small } \\
\text { molecule }\end{array}$ & $\begin{array}{c}\text { antineoplastic } \\
\text { antiangiogenesis }\end{array}$ & EGFR & $7.7-12.9$ & [26] \\
\hline Bevacizumab & Avastin & NSCLC & $\mathrm{rHMAb}$ & antiangiogenesis & VEGF & 6.2 & {$[28]$} \\
\hline
\end{tabular}

Table 1. Targeted therapies approved by the Food and Drug Administration in the treatment of lung cancer. The median time to progression on targeted therapy (Progression Free Survival - PFS) is given in months. rHMAb: recombinant human monoclonal antibody. Source: National Cancer Institute database, 2014.

\section{Lung cancer cell lines}

Cell lines derived from human tumors provide an unlimited, self-replicating source of malignant cells that can be studied by investigators throughout the world. Therefore, even if cell lines represent only a highly selected fraction of the original tumor, their ease of access has resulted in the production of a very large body of literature. Indeed, it is acknowledged that most of our understanding of the molecular mechanisms involved in LC comes from studies done on mouse or human cell lines [44]. 


\subsection{Lung cancer cell lines collection}

To date, more than 250 LC cell lines have been established, mainly from Western population. Currently, the American Type Culture Collection (ATCC, Manassas, VA) catalog lists 121 human lung tumor cell lines. Among this panel, SCLC is less represented, first due to the lower frequency of the disease, and second because SCLC tumors are rarely surgically resected. Indeed, only small tissue samples from biopsy examinations, malignant aspirates, and rare malignant effusions are available for research use. Moreover, the fact that SCLC tumor cells lack the ability to adhere to culture dishes and required to be grown in vitro as floating cell aggregates or spheroids has precluded for a long time their expansion as cell lines. SCLC was first successfully cultured in Japan in 1971 [45].

Regarding NSCLC, primary and metastatic tumor materials are more easily accessible, even through routine bronchoscopy [38, 39]. However, although cells from metastatic tumors, especially from malignant effusions, are relatively easy to culture, cell cultures from primary solid tumor are not obvious to establish, with success rates ranging from 2.6 to $5 \%$ [46, 47]. Various protocols are in use, but on the whole, tumor tissues minced in small pieces are either directly cultured as fragments in a matrix (e.g. Matrigel@) or subjected to enzymatic dissociation (collagenase/hyaluronidase) and then suspended in culture medium. Clearly, positive results are higher when starting from material corresponding to advanced stages as MHC III and IV [46]. The culture medium composition is also critical and the development of serumfree chemically defined media (e.g. ACL4) has significantly improved success rates [48].

The resulting current LC cell lines depository represent therefore a unique resource that can be extremely valuable in term of genetic manipulation, high-throughput screening and development of more complex co-culture models.

\subsection{Lung cancer cell lines as in vitro model}

LC cell lines have been used for decades in functional studies with the aim to identify new oncogene drivers or tumor suppressors. Thus, LC cell lines compared to normal human bronchial epithelial cells were instrumental to generate list of differentially expressed genes that could account for tumorigenicity and represent therefore new therapeutic targets. As an example, this strategy lead to the detection of $E R B B 3$, a gene associated with the EGF signaling pathway, among the genes over-expressed in LC cell lines [49]. Interestingly, this result was validated later on by another study that identified the activation of ERBB3 signaling as a mechanism of resistance to gefitinib [35]. Since these initial findings, ERBB3 has been recognized as a key node of LC progression and several humanized anti-ErbB3 antibodies are currently in pre-clinical development [50].

However, cell lines limitations have emerged as our knowledge about the disease increased. As an example, several studies have shown that differences in genetic background are important in defining cancer biology as well as in drug sensitivity [51]. Thus, a potential shortcoming of the current LC collection may reside in its under-representation of some populations, like the East Asian population, possibly introducing bias in research and drug discovery. Indeed, epidemiologic surveys have revealed that in the US, $10 \%$ of patients with NSCLC have tumour associated with EGFR mutations, while this number increases to $35 \%$ in 
East Asia, suggesting different selection mechanisms or sensitivity for lung cancer subtypes among different ethnic groups.

Accordingly, the recent classification of lung cancers into genetic subsets based on mutations in driver oncogenes (see previous section) prompted the community to accurately characterize the LC cell lines collection at the genomic and genetic levels. In this perspective, the Sanger Cancer Institute has initiated the genetic characterization of a panel of cancer cell lines (The Cancer Genome Project, http://cancer.sanger.ac.uk/cancergenome/projects/cell_lines/). Using current high throughput techniques this program provides information on mutations, copy number variations, single nucleotide polymorphisms (SNPs) and microsatellite instability of 136 cell lines representative of the different type of lung cancers (adenocarcinoma, small cell carcinoma, etc...) with the aim to define a genetic profile predictive of drug sensitivity. Such a signature should contribute to stratify patient population and to identify efficient targeted therapies.

Another emerging use of LC cell line is related to the identification of resistance mechanisms. As documented in section 2, so far all the approaches used in the treatment of lung cancer have resulted in the acquisition of resistance by the patients. One successful approach to discovering resistance mechanisms has been to culture sensitive cell lines with increasing concentrations of the drug until resistance emerges. The resistant cell line can subsequently be analysed to identify the resistance mechanisms, leading to the identification of resistance biomarkers and new strategies to overcome resistance [36].

Undoubtedly, cell lines have proven to be useful in elucidating important aspect of lung cancer biology. However, thanks to modern deep-sequencing technologies, we now know that lung tumors are composed of population of cells with distinct molecular and phenotypic properties [52] and consequently, that cell lines do not fully recapitulate human tumor biology. Clearly, the scientific community has taken into account these limitations, as shown by the growing interest for the establishment of complex in vitro cell models intended to bridge the gap between animal models and human studies.

\section{Biocompatible matrices for 3D cell culture}

In this section, we will try to briefly resume different ways and techniques used to culture the cells in 3D. Maintaining a 3D structure is critical to reproduce the tumour-stroma environment, communication between tumour cells, and the interaction with other surrounding cell types such as epithelial cells or fibroblasts [19]. Advances in materials chemistry and processing technologies, as well as developmental biology have led to the design of 3D cell culture matrices and bioscaffolds that better represent the geometry, chemistry, and signaling environment of natural extracellular matrix.

To obtain 3D cell cultures, cells are generally seeded onto/into biocompatible scaffolds or matrices. The 3D differentiation of cells depends on various parameters but it is generally accepted that best results are obtained when the natural environment is closely imitated [53]. Natural extracellular matrices are mainly composed of fibrous network made of collagen, elastic fibers, water and other materials like glycosaminoglycans, proteoglycans and glyco- 
proteins [54]. To mimic the natural extracellular environment of the cells important parameters have to be taken into account [55]:

1. The matrix composition (collagen, fibrin, alginate, etc.)

2. The structure (pore size, pore distribution, pore geometry, etc.)

3. The manufacturing method (electrospinning, 3D printing, inverted colloidal crystal, spontaneous polymerization, etc.)

4. The biocompatibility

As the fate of a cell is largely determined by its environment, the elaboration of the right extracellular context is critical to promote the correct differentiation of a cell population.

For example, it is well known that epithelial cells have to be cultured at the air-liquid interface to differentiate. This could be easily obtained by seeding cells onto micro porous supports or scaffolds allowing nutrients to come from the back. The apical side of the cells remains generally exposed to the air [56]. This basic principle of air-liquid interface cultivation can be transposed to most of epithelial cells such as airway, vaginal, buccal, intestinal, etc. However, this approach is no more suitable when the cells are not from epithelial origin $[57,58]$.

It is typically the case for fibroblasts that are not able to survive when directly exposed to the air. To culture fibroblasts in 3D, a different type of environment is required. Cells can be embedded into a biocompatible matrix based on various components [53]. Among the most used we find collagen and fibrin. Collagen is the major component of connective tissues, it is naturally produced by fibroblasts and can be easily isolated from many type of tissues such as dermis, bone, tendon, etc...

Whereas collagen is easily obtained, applications for human therapies and 3D cell culture remain limited because of contamination risks between animals and humans (e.g. CreutzfeldtJakob). Moreover, the gel polymerization can be difficult to control thus reducing the field of applicability. Another drawback is the variation between batches to batches. These weaknesses have led to the development of new generations of synthetic matrices and scaffolds where polymerization as well as intrinsic properties of materials (elasticity, porosity, permeability, hydration, etc.) can be more easily controlled. The matrices used today for 3D cell culture can be divided into 3 groups:

a. Natural compounds: collagen, gelatin, hyaluronate, glycosaminoglycan, chitosan, alginate, silk, fibrin, dextran, matrige $\AA^{\circledR}$, etc...

b. Synthetic compounds: polyglycolic acid (PGA), polylactic acid (PLA), polylactic-coglycolic acid (PLGA), polycarpolactone (PCL), polyethylene glycol (PEG), polyvinyl alcohol (PVA), polypropylene fumarate (PPF), polyacrylic acid (PAA), etc...

c. Mixed compounds, made of natural compounds synthetically modified. These included peptide-coupled alginates, chitosan, hyluranan, tyrosine-derived polymers, etc...

Each material has its own strengths and weaknesses and therefore it is fundamental to select matrix components in function of the needs. In the context of lung tumor cell model, it is 
pertinent to determine the final end-point studied. For example, if cells invasion has to be studied, it is necessary to use matrix components where cells are able to adhere, migrate and proliferate [59]. Moreover, some cells have the ability to digest and transform the scaffold and elaborate a new environment adapted to their needs. In that case it will be valuable to select a natural matrix component like collagen or fibrin. If the goal of the experiment is to obtain a 3D scaffold for human therapy, the best choice will be the use of synthetic matrices where all components can be defined and controlled [60]. If the sensitivity of a cancer cell to a drug has to be studied, a relevant choice could be the use of cell spheroids embedded into a non degradable matrix component, like alginate [61]. In that case, cells are immobilized and their drug susceptibility can be determined using a simple viability test. Alginate scaffold has been optimized for 3D tumor modeling using H460, A549 and H1650 NSCLC cell lines [62]. In this study the anticancer effects of various chemotherapeutic agents were studied and compared with conventional 2D cell culture models. Results have shown that cells grown in 3D demonstrated a more realistic drug response with higher resistance to chemotherapy [62].

Clearly, there is a tremendous flexibility to reconstitute a scaffold and the choice of synthesis should be guided by the type of cells, the application and the desired physical properties [53]. In addition, new perspectives are offered by bioprinting technologies. The possibility to organize extracellular matrix into precise geometries should help engineering 3D complex tumor tissues for in vitro assays [63].

\section{Tumor spheroid models for lung cancer research}

Numerous anchorage-independent assays have been developed for drug discovery. The most popular is the spheroid model because it allows both 3D self-organization of tumor cells and drug screening in high-throughput format. Many normal and malignant cell types can be grown as sphere-shaped cell colonies, so called spheroids. Cells that don't form spheroids spontaneously can be induced to do so by co-culturing with spheroid-forming non-clonogenic feeder cells [64]. As spheroid environment can be controlled, effects on tumor cell viability can be carefully examined. This model is particularly adapted to high throughput screening in 96well plate assays, and numerous solutions are commercially available.

Phenotypic and functional differences between lung tumor cells grown as 2D monolayer cultures, versus cells grown as 3D spheroids have been observed. Indeed, the 3D spheroid culture changed the cellular response to drugs and growth factors suggesting to be more accurately mimicking the natural tumor microenvironment than classical culture of lung cell line [65]. Multi-cell type tumor spheroids are a valuable model to reproduce cellular heterogeneity and provide more comprehensive assessment of tumor response to therapeutic strategies. 3D co-culture model using NSCLC cell lines in combination with lung fibroblasts can be prepared [66]. To date, co-cultures involving up to three different cell types in a single spheroid (tumor cells, fibroblasts and endothelial cells) have been established, but without any proof of micro-capillary functionality [67]. Recent studies report that NSCLC can acquire epithelial-mesenchymal transition and cancer stem-like phenotypes within chitosan-hyaluronan membrane-derived 3D tumor spheroids [68]. 


\section{Microfluidic chip-based 3D co-cultures}

In the continuity of the pioneering work of Donald Ingber (organ on chip), a series of 3D lungon-a-chip microfluidic devices have been developed. Briefly, lung-on-a-chip is a biomimetic microsystem that reconstitutes the critical functional alveolar-capillary interface of the human lung, with periodic mechanical stretching and flow of the medium carrying immune cells. Using this micro-fluidic device, the authors were able to replicating the immune responses against bacterial infections in vitro [69]. Afterwards, devices were optimized as a drug screening platform to select individualized treatment for lung cancer. In these systems, lung cancer and stromal cell lines were co-cultured as 3D spheroids under continuous media supplementation, mimicking the circulation of nutrients and metabolic waste out of the cultures [70]. Another similar model has been developed for chemoresistive testing of pleural mesothelioma cancer spheroids. Interestingly, growth inhibitory concentration of cisplatin showed higher concentration in perfused tumor spheroids compared with spheroids cultured under static conditions [71]. These systems represent therefore valuable tools to get information about the efficacy of chemotherapeutic drugs in a dynamic microenvironment which recapitulate the actual in vivo situation, but they do not address side-toxicity on normal lung physiology. The challenge will be to improve the model so that it incorporates normal and functional tissues. That could be achieved by connecting such devices with other microphysiological organotypic chips, representative of healthy lung tissues.

\section{Ex vivo 3D lung cancer model based on decellularized matrix}

As it is not obvious to identify the ideal matrix components and conditions suitable for the development of various lung tumor types, an alternative strategy is to take advantage of existing natural matrices. This methodology relies on the initial work of Ott and colleagues that first succeed in regenerating a bioartificial organ from a rat cadaveric lung [72]. Briefly, in this model the organ of interest is perfused with a detergent in order to remove donor cells and leave the components of the extracellular matrix. The resulting decellularized matrix is then reloaded with human lung adenocarcinoma tumor cells. In addition to their well-adapted composition, decellularized matrices also display specific elasticity which has been pointed out as critical for tumor cell growth. To date, rat decellularized lung matrix [73] and porcine decellularized intestinal submucosa [17] have been used as scaffold. Interestingly, tumor cell lines (A549, H460 and H1299) engrafted in this microenvironment formed 3D lung tumor nodules and displayed histological features reminiscent of the original primary tumor [74]. They also recovered functionalities (e.g. MMP-9 production) that were lost in 2D culture [73]. These ex vivo 3D models can be kept in culture for up to 28 days and exhibit sensitivity to treatment comparable to what is observed in clinic [17]. Although relevant for fundamental research, current ex vivo 3D lung models clearly show limitations. First, they are difficult to produce, the cultivation of the cells must take place in a special incubator, and consequently they cannot be used for high-throughput screening. Second, they do not recapitulate the 
human - human interactions between tumor and stroma. Indeed, epithelial and mesenchymal cells have been removed from epithelial space by the decellularization process. Third, they necessitate large amount of tumor cells ( $\sim 25$ millions) in order to colonize the matrix, precluding personalized medicine. And finally, they required the sacrifice of animals for matrix supply. However, ex vivo 3D lung models must be seen as the gold standard to be reached by 3D bioprinting technologies combined to synthetic matrices.

\section{Precision-cut lung tumor slices}

As previously mentioned, the tumor microenvironment provides essential signaling necessary for establishing and maintaining tumor specific morphogenic programs. Precision-cut lung slices (PCLS) obtained from freshly isolated human lung cancer tissues maintain both the original cancer microenvironment and preserve the complexity of the tumor-stroma interaction $[75,76]$. Usually thin tissue slices $(\sim 200 \mu \mathrm{M})$ are prepared with a vibratome and cultured submerged into medium for several days. PCLS constitute a valid tool for the in vitro evaluation of tumor morphology, proliferation, viability and resistance to therapy [75]. Moreover, a major advantage of this model is the preparation of multiple experimental replicates from a single tumor, allowing performing drug efficacy studies. Indeed, dose-response experiments with the PIK3 inhibitor LY294002 have shown that PCLS cultures from lung cancer may be used to predict tumor sensitivity to drugs in a patient-specific manner [75]. In a different study, tumor PCLS were used to investigate nanoparticles delivery of antisense as lung cancer treatment. The model was instrumental to demonstrate that nanoparticles could penetrate into tumor tissue and target telomerase activity, without disturbing adjacent tissue architecture or inducing significant side-toxicity [76]. PCLS established from human lung tumor tissue represent therefore a useful in vitro tumor model that has the potential to enhance preclinical drug evaluation studies. However, an obvious limitation of PCLS is their short lifespan, 5 days, which prevents long-term exposure, and therefore chronic treatment evaluation.

\section{Engineered 3D lung tumor tissues: The OncoCilAir ${ }^{\mathrm{TM}}$ model}

Tissue engineering is an innovative technology designed at first to produce artificial functional tissues to repair or replace portion of injured tissues. While initially seen as unrealistic, this field has made tremendous progress over the past decade, and regenerative medicine will soon become a routine technique [77]. Today, it is possible by combining human cells with suitable bioscaffolds to produce in vitro tissue equivalents from many sources (e.g. corneal, cartilage, intestinal, muscle, respiratory, skin, etc...). More recently, this promising technology has been applied to the field of oncology with some attempts to develop engineered tumor tissues for pre-clinical research (e.g. human melanoma model) [78]. Here we took advantage of our tissue engineering know-how in the respiratory field [79] to develop a complex, but accessible, 3D lung cancer model: OncoCilAir ${ }^{\mathrm{TM}}[80,81]$. To this purpose, human primary bronchial cells, 
lung fibroblasts and lung adenocarcinoma cell lines were co-cultured at the air-liquid interface in a transwell insert (Fig.2). After appropriate differentiation, the system closely reproduces malignant pulmonary nodules invading a human functional airway epithelium (Fig.3).

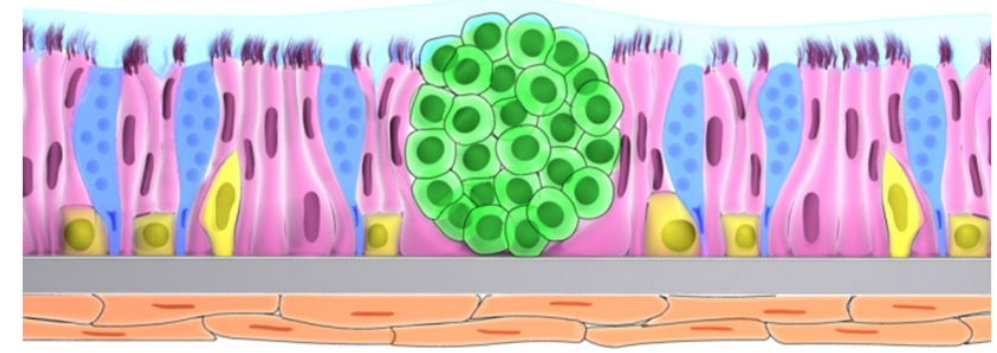

Figure 2. Schematic representation of the OncoCilAir ${ }^{\mathrm{TM}}$ lung cancer model. OncoCilAir ${ }^{\mathrm{TM}}$ is a complex cellular model based on the co-culture at the air-liquid-interface of three different human components: bronchial cells, lung fibroblasts and NSCLC cell lines. After 30 days, the cells differentiate into a functional respiratory epithelium which comprises ciliated cells (pink), goblet cells (blue) secreting mucus (light blue), basal cells (yellow), fibroblasts (brown) and tumor nodules (green).
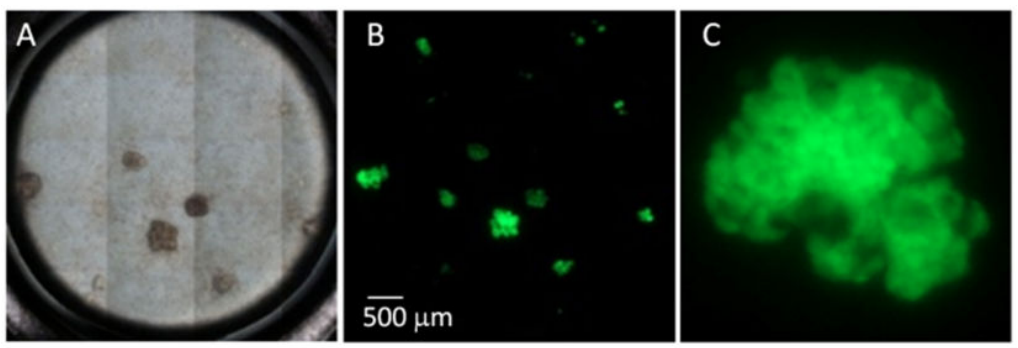

Figure 3. Cultured at the air-liquid interface in a convenient 24-wells format (A), the OncoCilAir ${ }^{\mathrm{TM}}$ model mimics the in vivo lung tissue of a patient with characteristic tumour lung nodules (B \& magnification in $\mathrm{C}$ ).

Several properties contribute to make OncoCilAir ${ }^{\mathrm{TM}}$ a relevant pre-clinical in vitro alternative: First, it is a $100 \%$ human three-dimensional model which summarizes human tumour-stroma interactions to assess therapies targeting host-tumor interactions (Fig.4). Second, it is a flexible system: depending on the cell line used to build its tumour component, OncoCilAir ${ }^{\mathrm{TM}}$ offers the possibility to recapitulate distinct molecular subsets of lung cancers (EGFR, KRAS, etc...) and thus to simulate patient stratification. Third, it is a bi-competency model: the fact that it includes both compromised and healthy tissues brings the possibility to experiment simultaneously drug efficacy and drug side-effect within a single culture. Lastly, its long lifespan (>3 months) allows to test chronic treatments and recurrence while reducing animal testing. 


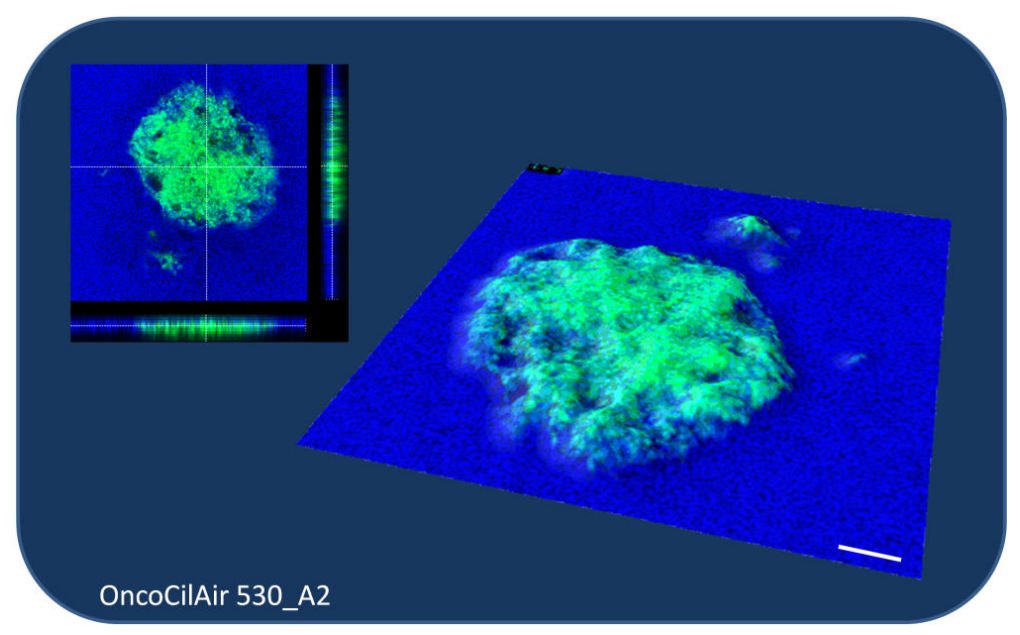

Figure 4. A tumor nodule expanding within the OncoCilAir ${ }^{\mathrm{TM}}$ human airway epithelium. Adenocarcima cells GFP+ (green) and human bronchial epithelial cells nuclei DAPI+ (blue) were visualized by confocal laser scanning microscopy. Scale bar represents $100 \mu \mathrm{m}$.

Accordingly, a dose response efficacy study with the investigational MEK inhibitors selumetinib and trametinib demonstrated that OncoCilAir ${ }^{\mathrm{TM}}$ showed responsiveness to anticancer drugs in agreement with previously reported data, and therefore can be used as a predictive tool for anticancer drug evaluation [82].

\section{Future perspective}

Hanahans and Weinberg highlighted six cancer hallmarks which provide us with a framework to understand this complex disease. These hallmarks include (i) sustaining proliferative signaling, (ii) evading growth suppressors, (iii) resisting cell death (iv) enabling replicative immortality, (v) inducing angiogenesis, and (vi) activating invasion and metastasis [83, 84]. However, in essence, cancer is a genetic disease with germ or autosomal mutations affecting genes implicated in cell division and/or in tissue integrity. These genetic alterations lead to unrestricted cell division and formation of a clone of cells which undergo further genetic changes. Some of these mutations promote features that endow cells with a selective advantage over normal cells, thus creating a more aggressive subclone with an even higher mutation rate, eventually leading to tumor formation [85]. The clonal theory has been corroborated by several decades of cancer researches: we now know that mutation in some specific genes, so-called oncogenes and tumor supressors, are primary cause for cancers. For lung cancers, the components of EGF signaling, such as EGF receptor and its downstream effectors (KRAS, BRAF, ALK, etc...) are the main drivers [33]. With the advance in biotechnology, it is now possible to rapidly identify the underlying mutations of the lung cancers for diagnostics and for personalized treatment. 


\begin{tabular}{|c|c|c|}
\hline $\begin{array}{c}\text { Cancer } \\
\text { hallmarks }\end{array}$ & Barriers & $\begin{array}{c}\text { Appropiate } \\
\text { in vitro models }\end{array}$ \\
\hline $\begin{array}{l}\text { Sustaining } \\
\text { proliferative } \\
\text { signal }\end{array}$ & $\begin{array}{l}\text { Inadequate } \\
\text { growth } \\
\text { promotion }\end{array}$ & \multirow{4}{*}{$\begin{array}{c}\text { Cell lines } \\
\text { Spheroids } \\
\text { 3D co-cultures } \\
\text { in vitro PnP }\end{array}$} \\
\hline $\begin{array}{l}\text { Evading growth } \\
\text { suppressors }\end{array}$ & & \\
\hline $\begin{array}{l}\text { Resisting } \\
\text { cell death }\end{array}$ & $\begin{array}{c}\text { Apoptosis with } \\
\text { loss of } \\
\text { basement } \\
\text { membrane }\end{array}$ & \\
\hline $\begin{array}{l}\text { Enabling } \\
\text { replicative } \\
\text { immortality }\end{array}$ & Senescence & \\
\hline $\begin{array}{l}\text { Inducing } \\
\text { angiogenesis }\end{array}$ & Ischaemia & \multirow{2}{*}{$\begin{array}{l}\text { 3D co-cultures } \\
\text { in vitro } \mathrm{PnP}\end{array}$} \\
\hline $\begin{array}{l}\text { Activating } \\
\text { invasion and } \\
\text { metastasis }\end{array}$ & $\begin{array}{l}\text { ECM and } \\
\text { epithelial } \\
\text { barrier }\end{array}$ & \\
\hline $\begin{array}{l}\text { Deregulating } \\
\text { cellular } \\
\text { energetics }\end{array}$ & Acidosis & in vitro $\mathrm{PnP}$ \\
\hline
\end{tabular}

Table 2. Current and future in vitro lung cancer models sorted according to cancer hallmarks.

However, a genetic change is only one side of the same coin. It is generally recognized now that the microenvironment surrounding the cancer cells plays also a crucial role in cancer development $[19,83,84]$. Indeed, tumor cells have to overcome at least six barriers in order to become invasive [86]. The extracellular matrix, stroma cells, immune cells, etc... form an integral part of the tumor, therefore should be taken into account. In fact, not all the cancer cells can grow in standard cell culture conditions: out of 160 tumors, only 8 Chinese NSCLC cell lines have been established in culture [47].

Therefore, for tissue engineering, the most important, as well as the most challenging task is to recreate the in vivo-like tumor micro-environment.

Another important issue is to maintain the heterogeneity of the tumor populations. Despite of huge progresses made in cancer research, the toll cancer claims in both human lives and funds spent on health care has been only marginally reduced, and in some cases even increases [87, 88]. One of the reasons for this situation is the drug resistance. The underlying cause is the heterogeneity of the tumor cells: within a tumor several clones with different mutations may co-exist. Furthermore, another process termed the community effect may be involved. Studies have suggested that the ability of a cell to respond to a signal may be enhanced by, or even dependent on, other neighboring cells reacting in the same way at the same time [89]. This effect helps to explain the formation of blocks of tissue from sheets of cells, and could be of widespread occurrence and significance in various morphogenesis processes, including tumor development. The underlying mechanism of the community effect could be the autocrine or paracrine positive feedback loops, which have also been suggested and identified during tumor formation. Several studies have outlined the importance of autocrine IL-6 signaling in lung and breast cancers. For example, one group found a positive correlation between 
persistently activated tyrosine-phosphorylated STAT3, found in 50\% of lung adenocarcinomas, and IL-6. Further investigation revealed that mutant EGFR could activate the oncogenic STAT3 pathway via upregulated IL-6 autocrine signaling [90].

The fact that most of the cancer cells, even the aggressive ones, cannot grow in culture once dissociated strongly supports this notion. In other words, all the cancer hallmarks are the hallmarks of the tumor as a whole, not that of individual cancer cells. This has to be taken into account during the development of in vitro cancer models.

\begin{tabular}{|c|c|c|c|c|}
\hline & Cell lines & Spheroids & $\begin{array}{l}\text { 3D Co-culture } \\
\text { Models }\end{array}$ & In vitro $\mathrm{PnP}$ \\
\hline 음 & $\begin{array}{l}\text { Appropriate for } \\
\text { identifying the } \\
\text { mutations and for } \\
\text { elucidating the } \\
\text { signalling pathways. } \\
\text { High throughput } \\
\text { screening of drug } \\
\text { candidates. }\end{array}$ & 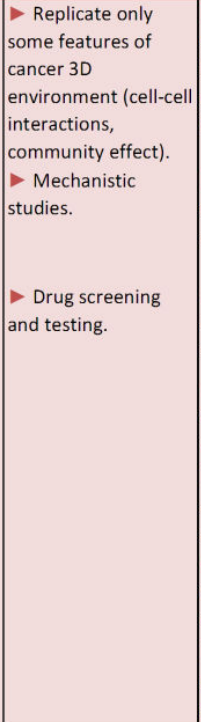 & $\begin{array}{l}\text { Reflect closely in } \\
\text { vivo situations, } \\
\text { having relevant cell- } \\
\text { cell and cell-stroma } \\
\text { interactions. } \\
\text { Possibility to study } \\
\text { cancer metastasis } \\
\text { and tumor invasion. } \\
\text { Allow testing the } \\
\text { efficacy and toxicity } \\
\text { simultaneously. } \\
\text { Allow chronic } \\
\text { treatments and } \\
\text { resistance studies. } \\
\text { Provide insight } \\
\text { about mechanistic at } \\
\text { all levels. } \\
\text { Identification and } \\
\text { validation of drug } \\
\text { candidate. } \\
\text { Suitable for } \\
\text { personalized } \\
\text { medicine. }\end{array}$ & $\begin{array}{l}\text { An ideal model } \\
\text { replicating most of the } \\
\text { features of cancer } \\
\text { development and } \\
\text { cancer growth. } \\
\text { Cell-cell and cell- } \\
\text { stroma interactions, } \\
\text { metastasis, immune } \\
\text { responses, drugs } \\
\text { circulation and } \\
\text { metabolization, } \\
\text { angiogenesis, etc... }\end{array}$ \\
\hline نั & $\begin{array}{l}\text { Do not reflect the } \\
\text { original in vivo } \\
\text { situations, lack of } \\
\text { relevant cell-cell and } \\
\text { cell-stroma } \\
\text { interactions. } \\
\text { loss of relevant } \\
\text { biomarkers. } \\
\text { Lack of mechanical } \\
\text { constraints from the } \\
\text { adjacent healthy tissue. }\end{array}$ & $\begin{array}{l}\text { Not cultured at ALI } \\
\text { condition, these } \\
\text { models are less } \\
\text { relevant for lung } \\
\text { cancers. } \\
\text { Not possible to } \\
\text { study metastasis. } \\
\text { Lack of immune } \\
\text { cells and no } \\
\text { angiogenesis. }\end{array}$ & $\begin{array}{l}\text { Lack of immune } \\
\text { cells and no } \\
\text { angiogenesis. }\end{array}$ & $\begin{array}{l}\text { Could be difficult } \\
\text { to standardize and to } \\
\text { scale-up. } \\
\text { Special expertise is } \\
\text { needed. Often patent- } \\
\text { protected. }\end{array}$ \\
\hline
\end{tabular}

Table 3. Strengths and weaknesses of the different human in vitro models for lung cancer.

Ideally, in vitro lung cancer models should recapture all the hallmarks of human lung cancer. Each model has its own strength and weaknesses (Table 3). But depending on the application, simple models may be more relevant and sufficient. Models can therefore be sorted by complexity: 
1. Cell lines

2. As simplest 3D model, the tumor spheroids represent already a progress with regarding to monolayer culture of tumor cell lines or primary tumor cells: the cell-cell interaction is restored. Stroma cells and or matrix could also be added to better mimic the in vivo situation.

3. Since the lung tumor cells are located at air-liquid interface, co-culture with the normal airway epithelial cells and fibroblast cells at air-liquid interface, illustrated by OncoCi$1 \mathrm{Air}^{\mathrm{TM}}$, is another realistic scenario for modeling the lung tumors.

4. In vitro PnP (Plug and Play) models with primary tumor derived from the patient.

In addition, Table 2 summarizes how the current, as well as the future in vitro cancer models can replicate some or all the cancer hallmarks, their use and limitations for drug development.

Finally, we would like to propose an ideal in vitro lung cancer model based on the above considerations, so-called in vitro PnP (Plug and Play) model (Fig.5).

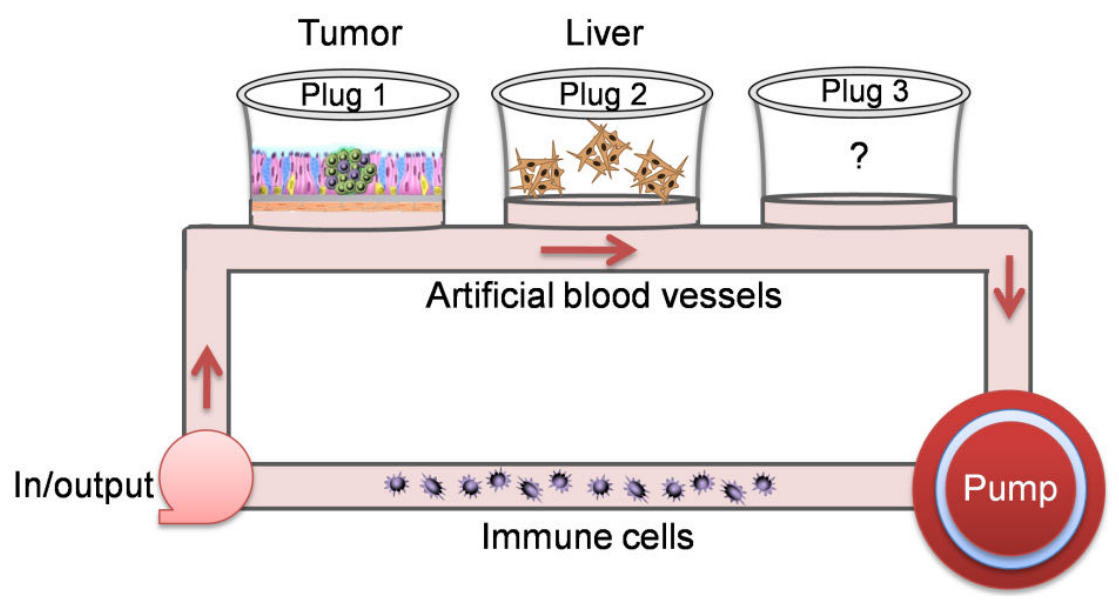

Figure 5. Schematic representation of an ideal in vitro PnP (Plug and Play) lung cancer model. A primary tumor derived from the patient is incorporated into a fully differentiated and healthy airway epithelium and cultured at airliquid interface in a setting similar to OncoCilair ${ }^{\mathrm{TM}}$ (Mas et al., 2015); Then this co-culture model is plugged into a micro-fluidic device with artificial blood/or lymphatic vessels (pink color) containing circulating immune cells (blue color); liver cells (hepatocytes as spheroids, brown color) can also be integrated into the circuit through the plug number 2, providing metabolic capacity of drugs. If needed, other cells/organs can be further inter-connected in similar way. An input/output plug allows the addition of drug or the uptake of medium for analysis. The lung tissue culture remains accessible to apical exposure during all the experiment.

A primary tumor derived from the patient is incorporated into a fully differentiated and healthy airway epithelium and cultured at air-liquid interface, a setting similar to OncoCilair ${ }^{\mathrm{TM}}$ [82]. Then this co-culture model is plugged into a micro-fluidic device with artificial blood/or lymphatic vessels containing circulating immune cells; liver cells (hepatocytes as spheroids) 
can also be plugged into the circuit, providing metabolic capacity of drugs. If needed, other cells/organs can be further inter-connected in a similar way.

We are convinced that, with the development of new technologies such as microfluidic devices and 3D bio-printing, such models should quickly emerge and strengthen in vitro pre-clinical cancer research.

\section{Author details}

Samuel Constant, Song Huang, Ludovic Wisniewski and Christophe Mas*

*Address all correspondence to: christophe.mas@oncotheis.com

OncoTheis Sàrl, Geneva, Switzerland

\section{References}

[1] Malvezzi, M., et al., European cancer mortality predictions for the year 2013. Ann Oncol, 2013. 24(3): p. 792-800.

[2] Arrowsmith, J. and P. Miller, Trial watch: phase II and phase III attrition rates 2011-2012. Nat Rev Drug Discov, 2013. 12(8): p. 569.

[3] Scannell, J.W., et al., Diagnosing the decline in pharmaceutical RED efficiency. Nat Rev Drug Discov, 2012. 11(3): p. 191-200.

[4] Rangarajan, A. and R.A. Weinberg, Opinion: Comparative biology of mouse versus human cells: modelling human cancer in mice. Nat Rev Cancer, 2003. 3(12): p. 952-9.

[5] Kung, A.L., Practices and pitfalls of mouse cancer models in drug discovery. Adv Cancer Res, 2007. 96: p. 191-212.

[6] Ruggeri, B.A., F. Camp, and S. Miknyoczki, Animal models of disease: pre-clinical animal models of cancer and their applications and utility in drug discovery. Biochem Pharmacol, 2014. 87(1): p. 150-61.

[7] Mak, I.W., N. Evaniew, and M. Ghert, Lost in translation: animal models and clinical trials in cancer treatment. Am J Transl Res, 2014. 6(2): p. 114-8.

[8] Odom, D.T., et al., Tissue-specific transcriptional regulation has diverged significantly between human and mouse. Nat Genet, 2007. 39(6): p. 730-2.

[9] Forsyth, N.R., W.E. Wright, and J.W. Shay, Telomerase and differentiation in multicellular organisms: turn it off, turn it on, and turn it off again. Differentiation, 2002. 69(4-5): p. 188-97. 
[10] Rangarajan, A., et al., Species- and cell type-specific requirements for cellular transformation. Cancer Cell, 2004. 6(2): p. 171-83.

[11] Seok, J., et al., Genomic responses in mouse models poorly mimic human inflammatory diseases. Proc Natl Acad Sci U S A, 2013. 110(9): p. 3507-12.

[12] Dorman, G., et al., Matrix metalloproteinase inhibitors: a critical appraisal of design principles and proposed therapeutic utility. Drugs, 2010. 70(8): p. 949-64.

[13] Dong, X., et al., Patient-derived first generation xenografts of non-small cell lung cancers: promising tools for predicting drug responses for personalized chemotherapy. Clin Cancer Res, 2010. 16(5): p. 1442-51.

[14] Moro, M., et al., Patient-derived xenografts of non small cell lung cancer: resurgence of an old model for investigation of modern concepts of tailored therapy and cancer stem cells. J Biomed Biotechnol, 2012. 2012: p. 568567.

[15] Rong, S., et al., Tumorigenicity of the met proto-oncogene and the gene for hepatocyte growth factor. Mol Cell Biol, 1992. 12(11): p. 5152-8.

[16] Mestas, J. and C.C. Hughes, Of mice and not men: differences between mouse and human immunology. J Immunol, 2004. 172(5): p. 2731-8.

[17] Stratmann, A.T., et al., Establishment of a human 3D lung cancer model based on a biological tissue matrix combined with a Boolean in silico model. Mol Oncol, 2014. 8(2): p. 351-65.

[18] Lee, J.J., et al., Stromal response to Hedgehog signaling restrains pancreatic cancer progression. Proc Natl Acad Sci U S A, 2014. 111(30): p. E3091-100.

[19] Egeblad, M., E.S. Nakasone, and Z. Werb, Tumors as organs: complex tissues that interface with the entire organism. Dev Cell, 2010. 18(6): p. 884-901.

[20] Chen, W.J., et al., Cancer-associated fibroblasts regulate the plasticity of lung cancer stemness via paracrine signalling. Nat Commun, 2014. 5: p. 3472.

[21] Malvezzi, M., et al., European cancer mortality predictions for the year 2014. Ann Oncol, 2014. 25(8): p. 1650-6.

[22] Pesch, B., et al., Cigarette smoking and lung cancer--relative risk estimates for the major histological types from a pooled analysis of case-control studies. Int J Cancer, 2012. 131(5): p. $1210-9$.

[23] Brambilla, E., et al., The new World Health Organization classification of lung tumours. Eur Respir J, 2001. 18(6): p. 1059-68.

[24] Ohe, Y., et al., Randomized phase III study of cisplatin plus irinotecan versus carboplatin plus paclitaxel, cisplatin plus gemcitabine, and cisplatin plus vinorelbine for advanced nonsmall-cell lung cancer: Four-Arm Cooperative Study in Japan. Ann Oncol, 2007. 18(2): p. 317-23. 
[25] Reck, M., et al., Tissue sampling in lung cancer: a review in light of the MERIT experience. Lung Cancer, 2011. 74(1): p. 1-6.

[26] Nguyen, K.S., S. Kobayashi, and D.B. Costa, Acquired resistance to epidermal growth factor receptor tyrosine kinase inhibitors in non-small-cell lung cancers dependent on the epidermal growth factor receptor pathway. Clin Lung Cancer, 2009. 10(4): p. 281-9.

[27] Rosell, R., et al., Screening for epidermal growth factor receptor mutations in lung cancer. N Engl J Med, 2009. 361(10): p. 958-67.

[28] Sandler, A., et al., Paclitaxel-carboplatin alone or with bevacizumab for non-small-cell lung cancer. N Engl J Med, 2006. 355(24): p. 2542-50.

[29] Camidge, D.R., et al., Activity and safety of crizotinib in patients with ALK-positive nonsmall-cell lung cancer: updated results from a phase 1 study. Lancet Oncol, 2012. 13(10): p. 1011-9.

[30] Doebele, R.C., et al., Mechanisms of resistance to crizotinib in patients with ALK gene rearranged non-small cell lung cancer. Clin Cancer Res, 2012. 18(5): p. 1472-82.

[31] Shaw, A.T., et al., Ceritinib in ALK-rearranged non-small-cell lung cancer. N Engl J Med, 2014. 370(13): p. 1189-97.

[32] Chen, X., et al., Clinical perspective of afatinib in non-small cell lung cancer. Lung Cancer, 2013. 81(2): p. 155-61.

[33] Tsvetkova, E. and G.D. Goss, Drug resistance and its significance for treatment decisions in non-small-cell lung cancer. Curr Oncol, 2012. 19(Suppl 1): p. S45-51.

[34] Pao, W., et al., Acquired resistance of lung adenocarcinomas to gefitinib or erlotinib is associated with a second mutation in the EGFR kinase domain. PLoS Med, 2005. 2(3): p. e73.

[35] Engelman, J.A., et al., MET amplification leads to gefitinib resistance in lung cancer by activating ERBB3 signaling. Science, 2007. 316(5827): p. 1039-43.

[36] Katayama, R., et al., Mechanisms of acquired crizotinib resistance in ALK-rearranged lung Cancers. Sci Transl Med, 2012. 4(120): p. 120ra17.

[37] Ogawa, T., et al., Methylation of death-associated protein kinase is associated with cetuximab and erlotinib resistance. Cell Cycle, 2012. 11(8): p. 1656-63.

[38] Sequist, L.V., et al., Genotypic and histological evolution of lung cancers acquiring resistance to EGFR inhibitors. Sci Transl Med, 2011.3(75): p. 75ra26.

[39] Yu, H.A., et al., Analysis of tumor specimens at the time of acquired resistance to EGFR-TKI therapy in 155 patients with EGFR-mutant lung cancers. Clin Cancer Res, 2013. 19(8): p. 2240-7.

[40] Mintz, B. and K. Illmensee, Normal genetically mosaic mice produced from malignant teratocarcinoma cells. Proc Natl Acad Sci U S A, 1975. 72(9): p. 3585-9. 
[41] Mitra, A.K., et al., MicroRNAs reprogram normal fibroblasts into cancer-associated fibroblasts in ovarian cancer. Cancer Discov, 2012. 2(12): p. 1100-8.

[42] Sherman, M.H., et al., Vitamin d receptor-mediated stromal reprogramming suppresses pancreatitis and enhances pancreatic cancer therapy. Cell, 2014. 159(1): p. 80-93.

[43] Rowley, D.R., Reprogramming the tumor stroma: a new paradigm. Cancer Cell, 2014. 26(4): p. 451-2.

[44] Gazdar, A.F., et al., Lung cancer cell lines as tools for biomedical discovery and research. J Natl Cancer Inst, 2010. 102(17): p. 1310-21.

[45] Oboshi, S., et al., A new floating cell line derived from human pulmonary carcinoma of oat cell type. Gan, 1971. 62(6): p. 505-14.

[46] Sugaya, M., et al., Establishment of 15 cancer cell lines from patients with lung cancer and the potential tools for immunotherapy. Chest, 2002. 122(1): p. 282-8.

[47] Zheng, C., et al., Establishment and characterization of primary lung cancer cell lines from Chinese population. Acta Pharmacol Sin, 2011. 32(3): p. 385-92.

[48] Gazdar, A.F. and H.K. Oie, Cell culture methods for human lung cancer. Cancer Genet Cytogenet, 1986. 19(1-2): p. 5-10.

[49] Lam, D.C., et al., Establishment and expression profiling of new lung cancer cell lines from Chinese smokers and lifetime never-smokers. J Thorac Oncol, 2006. 1(9): p. 932-42.

[50] Aurisicchio, L., et al., The promise of anti-ErbB3 monoclonals as new cancer therapeutics. Oncotarget, 2012. 3(8): p. 744-58.

[51] Sekine, I., et al., Emerging ethnic differences in lung cancer therapy. Br J Cancer, 2008. 99(11): p. 1757-62.

[52] Zhang, J., et al., Intratumor heterogeneity in localized lung adenocarcinomas delineated by multiregion sequencing. Science, 2014. 346(6206): p. 256-9.

[53] Lee, J., M.J. Cuddihy, and N.A. Kotov, Three-dimensional cell culture matrices: state of the art. Tissue Eng Part B Rev, 2008. 14(1): p. 61-86.

[54] Matsusaki, M., C.P. Case, and M. Akashi, Three-dimensional cell culture technique and pathophysiology. Adv Drug Deliv Rev, 2014. 74: p. 95-103.

[55] Sant, S., et al., Biomimetic Gradient Hydrogels for Tissue Engineering. Can J Chem Eng, 2010. 88(6): p. 899-911.

[56] Margulis, A., W. Zhang, and J.A. Garlick, In vitro fabrication of engineered human skin. Methods Mol Biol, 2005. 289: p. 61-70.

[57] Huang, S., et al., Potential of in vitro reconstituted 3D human airway epithelia (MucilAir) to assess respiratory sensitizers. Toxicol In vitro, 2013. 27(3): p. 1151-6. 
[58] Schaller, M., et al., Models of oral and vaginal candidiasis based on in vitro reconstituted human epithelia. Nat Protoc, 2006. 1(6): p. 2767-73.

[59] Even-Ram, S. and K.M. Yamada, Cell migration in 3D matrix. Curr Opin Cell Biol, 2005. 17(5): p. 524-32.

[60] Vacanti, J.P. and R. Langer, Tissue engineering: the design and fabrication of living replacement devices for surgical reconstruction and transplantation. Lancet, 1999. 354 Suppl 1: p. SI32-4.

[61] Bohari, S.P., D.W. Hukins, and L.M. Grover, Effect of calcium alginate concentration on viability and proliferation of encapsulated fibroblasts. Biomed Mater Eng, 2011. 21(3): p. 159-70.

[62] Godugu, C., et al., AlgiMatrix based 3D cell culture system as an in-vitro tumor model for anticancer studies. PLoS One, 2013. 8(1): p. e53708.

[63] Zhao, Y., et al., Three-dimensional printing of Hela cells for cervical tumor model in vitro. Biofabrication, 2014. 6(3): p. 035001.

[64] Djordjevic, B. and C.S. Lange, Clonogenicity of mammalian cells in hybrid spheroids: a new assay method. Radiat Environ Biophys, 1990. 29(1): p. 31-46.

[65] Ekert, J.E., et al., Three-dimensional lung tumor microenvironment modulates therapeutic compound responsiveness in vitro--implication for drug development. PLoS One, 2014. 9(3): p. e92248.

[66] Amann, A., et al., Development of an innovative 3D cell culture system to study tumour-stroma interactions in non-small cell lung cancer cells. PLoS One, 2014. 9(3): p. e92511.

[67] Thoma, C.R., et al., 3D cell culture systems modeling tumor growth determinants in cancer target discovery. Adv Drug Deliv Rev, 2014. 69-70: p. 29-41.

[68] Huang, Y.J. and S.H. Hsu, Acquisition of epithelial-mesenchymal transition and cancer stem-like phenotypes within chitosan-hyaluronan membrane-derived 3D tumor spheroids. Biomaterials, 2014. 35(38): p. 10070-9.

[69] Huh, D., et al., Reconstituting organ-level lung functions on a chip. Science, 2010. 328(5986): p. 1662-8.

[70] Xu, Z., et al., Application of a microfluidic chip-based 3D co-culture to test drug sensitivity for individualized treatment of lung cancer. Biomaterials, 2013. 34(16): p. 4109-17.

[71] Ruppen, J., et al., A microfluidic platform for chemoresistive testing of multicellular pleural cancer spheroids. Lab Chip, 2014. 14(6): p. 1198-205.

[72] Ott, H.C., et al., Regeneration and orthotopic transplantation of a bioartificial lung. Nat Med, 2010. 16(8): p. 927-33.

[73] Mishra, D.K., et al., Human lung cancer cells grown on acellular rat lung matrix create perfusable tumor nodules. Ann Thorac Surg, 2012. 93(4): p. 1075-81. 
[74] Mishra, D.K., et al., Human lung cancer cells grown in an ex vivo 3D lung model produce matrix metalloproteinases not produced in 2D culture. PLoS One, 2012. 7(9): p. e45308.

[75] Vaira, V., et al., Preclinical model of organotypic culture for pharmacodynamic profiling of human tumors. Proc Natl Acad Sci U S A, 2010. 107(18): p. 8352-6.

[76] Dong, M., et al., Tissue slice model of human lung cancer to investigate telomerase inhibition by nanoparticle delivery of antisense 2'-O-methyl-RNA. Int J Pharm, 2011. 419(1-2): p. 33-42.

[77] Atala, A., F.K. Kasper, and A.G. Mikos, Engineering complex tissues. Sci Transl Med, 2012. 4(160): p. 160rv12.

[78] Walles, T., et al., The potential of bioartificial tissues in oncology research and treatment. Onkologie, 2007. 30(7): p. 388-94.

[79] Wiszniewski, L., et al., Long-term cultures of polarized airway epithelial cells from patients with cystic fibrosis. Am J Respir Cell Mol Biol, 2006. 34(1): p. 39-48.

[80] Mas C, Huang S, Wiszniewski L, Constant S., A new 3D human airway tissue model for in vitro lung cancer research (OncoCilAir ${ }^{\mathrm{TM}}$ ). ALTEX Proceedings, 2014. 3(1/14): p. I-4-204.

[81] Mas C, Huang S, Wiszniewski L, Constant S., In vitro Human Airway Tissue Model For Lung Cancer Research. Am J Respir Crit Care Med 2014. 189: p. A3455.

[82] Mas C, Boda B, CaulFuty M, Huang S, Wiszniewski L and Constant S., Antitumour efficacy of the selumetinib and trametinib MEK inhibitors in a combined human airway-tumour-stroma lung cancer model. J Biotechnol., 2015. in press.

[83] Hanahan, D. and R.A. Weinberg, The hallmarks of cancer. Cell, 2000. 100(1): p. 57-70.

[84] Hanahan, D. and R.A. Weinberg, Hallmarks of cancer: the next generation. Cell, 2011. 144(5): p. 646-74.

[85] Nowell, P.C., The clonal evolution of tumor cell populations. Science, 1976. 194(4260): p. 23-8.

[86] Gatenby, R.A. and R.J. Gillies, A microenvironmental model of carcinogenesis. Nat Rev Cancer, 2008. 8(1): p. 56-61.

[87] Siegel, R., et al., Cancer statistics, 2011: the impact of eliminating socioeconomic and racial disparities on premature cancer deaths. CA Cancer J Clin, 2011. 61(4): p. 212-36.

[88] Malakoff, D., Can treatment costs be tamed? Science, 2011. 331(6024): p. 1545-7.

[89] Gurdon, J.B., A community effect in animal development. Nature, 1988. 336(6201): p. 772-4.

[90] Gao, S.P., et al., Mutations in the EGFR kinase domain mediate STAT3 activation via IL-6 production in human lung adenocarcinomas. J Clin Invest, 2007. 117(12): p. 3846-56. 
